\title{
Simulation Optimization of the Filling Process of Vacuum Infusion Molding Process for Composite Yacht Hull
}

\author{
YANG Bo ${ }^{1, a}$, JIN Tianguo ${ }^{2, b}$ and WANG Shenglong ${ }^{3, c}$ \\ 1, 2, 3 School of Mechatronics Engineering, Harbin Institute of Technology, Harbin 150001, China \\ ayb861025@163.com, bjintg@hit.edu.cn, cwangshenglong115@163.com
}

Keywords: yacht; VIMP; manufacturability evaluation; optimization; numerical simulation

\begin{abstract}
Vacuum infusion molding process(VIMP) is widely used for manufacturing the composite yacht hull, but the process parameters are designed by experience or lots of experiments. In this paper, the filling process of VIMP for composite yacht hull was deeply analyzed based on numerical simulation technology. Firstly, we simulated the filling process of yacht hull under different ply schemes, evaluated the manufacturability of the ply scheme based on the simulation results. Secondly, we determined the best runner layout pattern by simulating the filling process under four different runner layout patterns. Lastly, we studied the optimization method for gate locating of VIMP, established the mathematical model for optimizing the gate location and obtained the concrete form of the object function after a series of simulations, then obtained the optimal gate location result. The simulations also showed that it is inadvisable to reduce the filling time just by adding the gate quantity.
\end{abstract}

\section{Introduction}

Composite yacht has become the major product in yacht industry because of its superiorities such as low weight and high strength[1,2]. VIMP is a very attractive composite manufacturing process since large structures such as fuselages and yacht hull can be fabricated in a cost effective manner. The quality of mold filling stage in VIMP depends on the ply design and the pouring system which include runner layout and gate location. However, the design of the process routinely involves a trial and error method which make manufacturing costs and development time difficult to estimate.

The numerical simulation technology is a feasible solution[3,4]. After simulating the mold filling process, the rationality of the ply scheme and the pouring system can be judged, then the process parameters can be optimized[5]. Ali Gokce and Suresh G. Advani[6,7] proposed a cascaded optimization algorithm for simultaneous gate and vent location optimization in the presence of race tracking. Y.C. Lam, G.A. Britton and D.S. Liu[8] optimized the gate locations using the standard deviation of filling time as the objective function. Jiang Shunliang[9] proposed an approach to optimize the gate locations of RTM treating the trapping volume as penalty.

It was observed that the current researches are mainly aimed at composite or process itself, there are few study articles from the angle of parts or final products, especially the yacht hull. In this paper, we systematically study the influence of ply design and pouring system on the quality of mold filling of VIMP for yacht hull. The numerical simulations are implemented to obtain the flow parameters such as flow front and filling time, then the manufacturability evaluation of the ply design and the optimization of the pouring system are studied based on the simulation results.

\section{Manufacturability evaluation of the hull ply design}

The preform of the yacht hull is infiltrated by the vertical flow in the preform. Because of the mechanics performance requirements of yacht hull, the number of layers on the side of hull is smaller than that on the hull bottom, so is the preform permeability distribution. Thus, the inconsonant resin flow front may appear and result in an air entrapment as shown in Fig.1. From the flow characteristics we can conduct that the bigger permeability gap between hull side and bottom, 
the easier to generate defects. From the above analysis, we can find that the permeability has a important influence on the appearance of air entrapment.

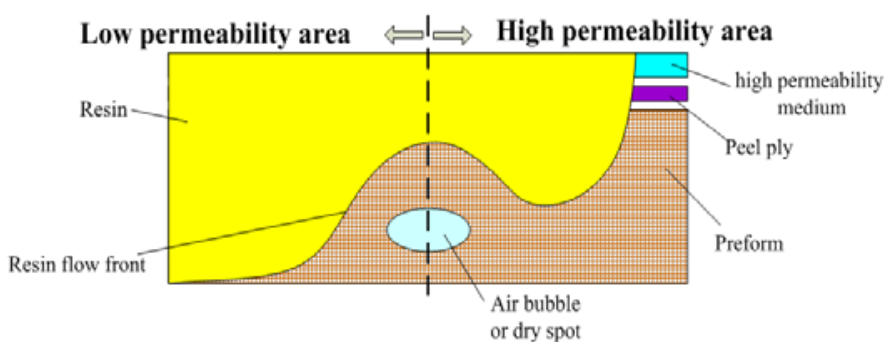

Fig.1 Air entrapment formation principle

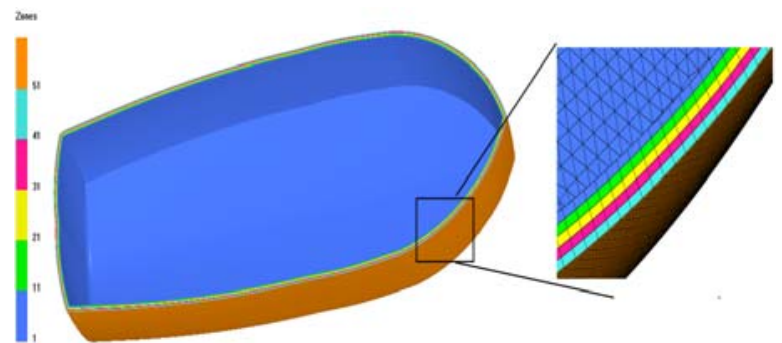

Fig.2 The analysis model of the tacht hull

The geometry parameters of yacht used in this paper are shown in Table 1. For simplifying the simulation procedure, we ignore the slight features and establish the geometry model of yacht hull for simulation as shown in Fig.2. All simulations in this paper are based on this model.

Table 1 Geometry parameters of the yacht hull

\begin{tabular}{cccc}
\hline Parameter name & Parameter value & Parameter name & Parameter value \\
\hline Length overall[m] & 12.19 & Molded depth[m] & 2.05 \\
Length of perpendiculars[m] & 10.60 & Draught[m] & 0.73 \\
Thickness[mm] & 8 & Molded breadth[m] & 4.10 \\
\hline
\end{tabular}

Using the reforcing material shown in Table 2 to ply the preform of the yacht hull, the ply shcemes are shown in Table 3.

Table 2 The properties of the reforcing material

\begin{tabular}{cccc}
\hline Material index & Material name & Thickness, $t[\mathrm{~mm}]$ & Density, $\rho\left[\mathrm{g} / \mathrm{cm}^{3}\right]$ \\
\hline 1 & Chopped Strand Mat & 0.7 & 1670 \\
2 & stitched mat & 1.5 & 1890 \\
3 & woven fabrics & 1 & 2670 \\
4 & Continuous fiber felt & 1.2 & 1760 \\
\hline
\end{tabular}

Table3 The ply schemes

\begin{tabular}{cccc}
\hline Scheme index & Ply property & Hull bottom & Hull side \\
\hline 1 & Stacking sequence & {$[1 / 3 / 1 / 2 / 3 / 1]$} & {$[1 / 2 / 3 / 1 / 2]$} \\
& Layer angle & {$[0 / 0 / 0 / 45 /-45 / 0]$} & {$[0 / 45 /-45 / 0 / 90]$} \\
& Permeability[m $\left.{ }^{2}\right]$ & $4.86 \times 10^{-10}$ & $5.25 \times 10^{-10}$ \\
2 & Stacking sequence & {$[1 / 4 / 1 / 4 / 2 / 1]$} & {$[1 / 2 / 4 / 1 / 3]$} \\
& Layer angle & {$[0 / 0 / 0 / 0 /-45 / 0]$} & {$[0 / 45 /-45 / 0 / 90]$} \\
& Permeability[m $\left.{ }^{2}\right]$ & $4.36 \times 10^{-10}$ & $5.34 \times 10^{-10}$ \\
\hline
\end{tabular}

For evaluating the manufacturability of the two ply schemes(Table 3 ) of the yacht hall, we predict the air entrapment by simulating the mold filling process. To this end, the geometry model is meshed and assigned material property as shown in Fig.2. The simulation results of the filling process are shown in Fig.3 and Fig.4.
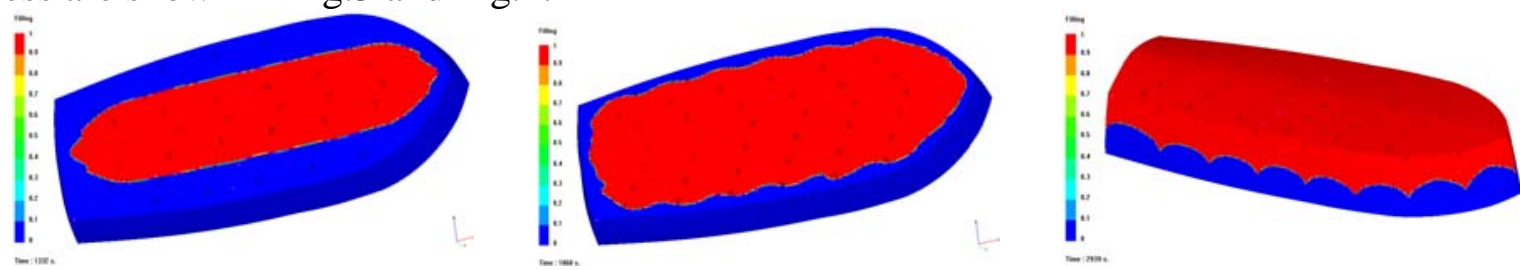

Fig.3 Filling process of ply scheme 1
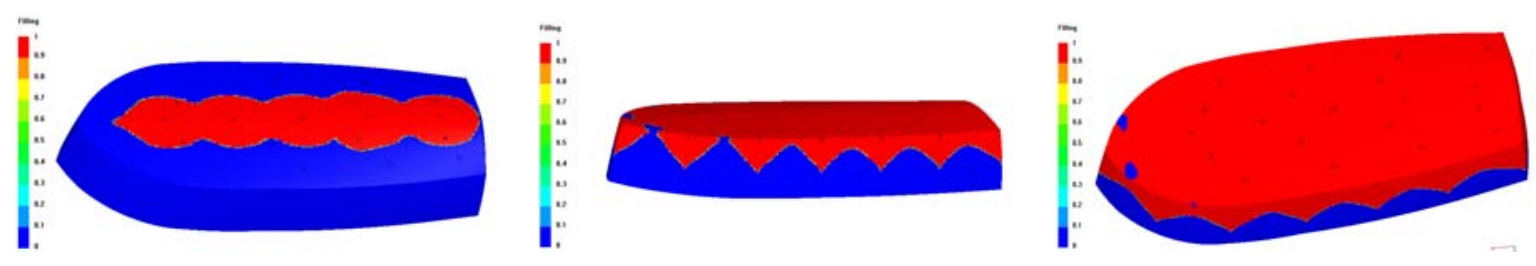

Fig.4 Filling process of ply scheme 2

Published by Atlantis Press, Paris, France.

(C) the authors 
From the Fig.3 and Fig.4, we can find that there are air entrapments appeared on the joint of the preform made by ply scheme 2. The simulation results show that the ply scheme with close permeability values on different regions of preform should be first chosen on the promise of guarantee of hull mechanics performance demands to avoid the appearance of air entrapment.

\section{Determination of the runner layout pattern}

Runner is the resin flow tube placed on the surface of the preform to accelerate the resin flow, the resin gates are evenly distributed on the runner. The runner layout patterns commonly used in industry are parallel layout pattern(Fig.5(a)) and fish-bone layout pattern(Fig.5(b)).

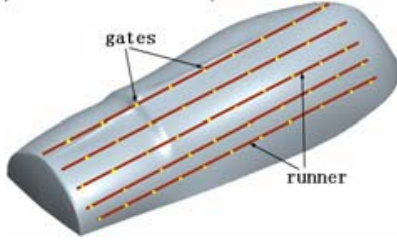

(a)

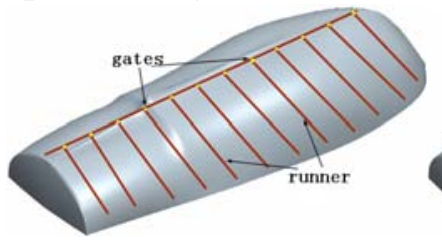

(b)

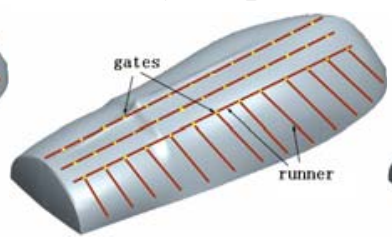

(c)

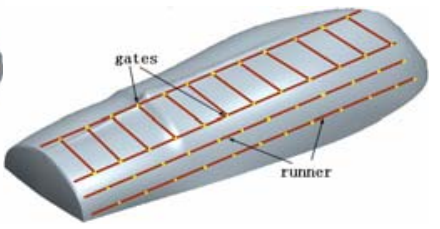

(d)

Fig.5 Runner layout patterns. (a) Parallel layout pattern; (b) Fish-bone layout pattern;

(c) Mixed-type 1 layout pattern; (d) Mixed-type 2 layout pattern.

The parallel layout pattern has good adaptability to complex geometry parts, but the resin flow velocity in mold is relatively low, filling time is long, so the resin curing reaction may occur during the filling process, it is really easy to cause the defects such as dry spot and incomplete filling. Although the resin flow velocity in mold with fish-bone runner layout is relatively high, the flow front is hard to control and lead to the air entrapment phenomenon mentioned in the above section. According to the above analysis, we propose two mix-type runner layout patterns based on the two existing patterns, named mixed-type 1 and mixed-type 2 layout patterns shown in Fig.5 (c) and (d).

Comprehensively considering the process cost and molding quality, we evaluate the runner layout pattern by mold filling time and flow front. For selecting the best runner layout pattern, we simulate the molding filling process under the above four runner layout patterns but the same hull geometry model and ply scheme, then decide the best one according to the simulation results. Because the preform is very thin, the $2 \mathrm{D}$ flow simulation is implemented instead of $3 \mathrm{D}$ simulation.

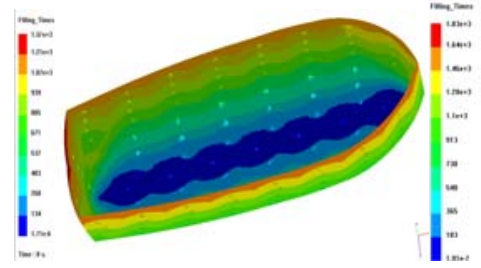

(a)

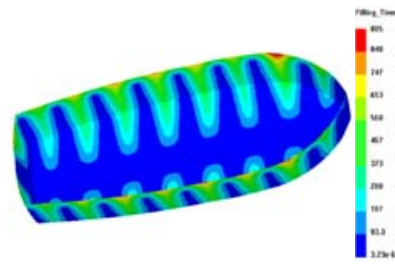

(b)

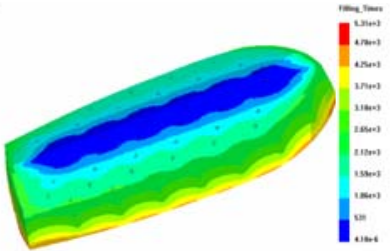

(c)

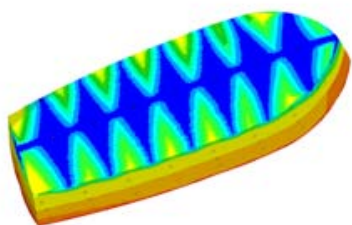

(d)

Fig.6 Simulation results under the four runner layout patterns. (a)Parallel layout pattern;

(b)Fish-bone layout pattern; (c)Mixed-type 1 layout pattern; (d) Mixed-type 2 layout pattern.

The simulation results are shown in Fig.6, we can find that the filling time of the four layout patterns are 1570s, 673s, 885s and 5310s. The flow front of mixed-type 2 layout pattern is seriously unreasonable, leads to a extremely long filling time. The flow front under the parallel layout pattern is fairly reasonable while the filling time is a little long, in addition, the operation and cost needs are much more than other patterns. The fish-bone pattern has the advantages of filling time and cost, but it's flow front is irrational and hard to control. Therefore, the mixed-type 1 layout pattern is the optimal selection because of its shorter filling time, lower costs and reasonable flow front.

\section{Optimization of the gate location}

In VIMP, the inlet pressure and outlet pressure are both constant, the pressure difference around the flow front reduces during the resin flow, so do the resin flow velocity. Obviously the more runners and gates, the better for decreasing the filling time and preform infiltration. However, excessive runners and gates need more materials and more operations for placing the tubes, more switch controlling and will cause more probability of defects. Hence, the minimum runners and 
gates should be used under the promise of filling quality. Optimization of the gate location is finding the optimal location and number of the resin gate to achieve the balance of filling time and process cost. The resin gates are evenly distributed on the runners as shown in Fig.7, $X$ denotes the distance between axial adjacent gates, $Y$ denotes the distance between radial adjacent gates.

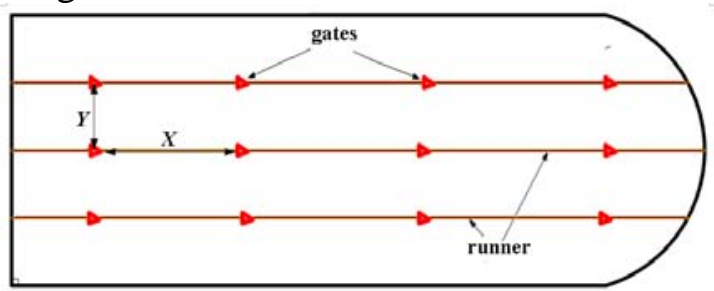

Fig.7 Resin gate location

The optimization objective function is the reflection of the influence of tuning parameters on the optimization objective. According to the above analysis, the mold filling time and process cost are employed to be the optimization objectives, note that the process cost include not only economic cost such as material and labor cost, but also operation complexity such as tube laying complexity and gate controling complexity, therefore, the process cost is a comprehensive value of process requirement. The tuning parameters are the location of the gate: $X$ and $Y$ as shown in Fig.7, so the following equations can be established:

$$
T=f_{1}(X, Y) ; C=f_{2}(\mathrm{X}, \mathrm{Y})
$$

here $T$ is the mold filling time, $C$ denotes the process cost, $f_{1}, f_{2}$ represent the functional relationship between the optimization objective and tuning parameters. The optimization objective function can be described by equation (2):

$$
\min O ; O=\phi_{T} T+\phi_{C} C=\phi_{T} f_{1}(X, Y)+\phi_{C} f_{2}(\mathrm{X}, \mathrm{Y})
$$

here $O$ is the final optimization objective, $\Phi_{T}$ is the weight of filling time, $\Phi_{C}$ is the weight of process cost.

The filling time is restricted by the resin gel time, process cost is restricted by the maximal acceptable cost:

$$
T<\varphi T_{g}, \varphi<1 ; C<C_{m}
$$

here $\phi$ denotes safety factor, commonly uses $0.8 ; T_{g}$ is the gel time of resin, set at 1800; $C_{m}$ is the maximal acceptable cost, set at 2000.

Since it is impossible to simulate the resin flow under all possible gate arrangements, we just implement the simulations under frequently-used gate arrangements as shown in Table 4 to deduce the concrete form of equations (1). The filling time simulation results are also listed in Table 4.

Table 4 The filling time of different gate arrangements

\begin{tabular}{cccccccc}
\hline$(X, Y)[\mathrm{mm}]$ & $\begin{array}{c}\text { Filling } \\
\text { time[s] }\end{array}$ & $(X, Y)[\mathrm{mm}]$ & $\begin{array}{c}\text { Filling time } \\
{[\mathrm{s}]}\end{array}$ & $(X, Y)[\mathrm{mm}]$ & $\begin{array}{c}\text { Filling time } \\
{[\mathrm{s}]}\end{array}$ & $(\mathrm{X}, Y)[\mathrm{mm}]$ & $\begin{array}{c}\text { Filling time } \\
{[\mathrm{s}]}\end{array}$ \\
\hline$(1500,400)$ & 885 & $(2000,400)$ & 993 & $(3000,400)$ & 1750 & $(2500,400)$ & 1320 \\
$(1500,500)$ & 1000 & $(2000,500)$ & 1230 & $(3000,500)$ & 1640 & $(2500,500)$ & 1420 \\
$(1500,600)$ & 1340 & $(2000,600)$ & 1530 & $(3000,600)$ & 1880 & $(2500,600)$ & 1740 \\
$(1500,800)$ & 1550 & $(2000,800)$ & 1630 & $(3000,800)$ & 2670 & $(2500,800)$ & 2150 \\
\hline
\end{tabular}

The relationship between gate number and filling time is plotted in Fig.8, the discrete points are the simulation results, the curve is the fitting curve of those points. We can find that the filling time extends sharply as the number of gate lessens, while as the number of gate increases, the reducing of the filling time becomes more and more slowly. Therefore, it is unconspicuous to reduce the filling time just by adding the gate quantity.

The fuction relation between tuning parameters and filling time can be confirmed by data fitting:

$$
T=-3.85 \times 10^{-5}-0.6 X+3.34 Y+2.25 \times 10^{-4} X Y+2.23 \times 10^{-4} X^{2}-1.47 \times 10^{-3} Y^{2}
$$

According to the yacht used in this paper and industrial actual process cost estimation method, the fuction relation between tuning parameters and process cost can be depicted by equation (5): 


$$
C=\frac{8.64 \times 10^{8}}{X Y}+\frac{3.792 \times 10^{5}}{X}+\frac{7.2 \times 10^{4}}{Y}+78
$$

Equations (4) and (5) are the concrete form of equations (1). Assuming the weight of filling time and process cost are equal, we can obtain the relationship between optimization objective and tuning parameters and draw it on Fig. 9.

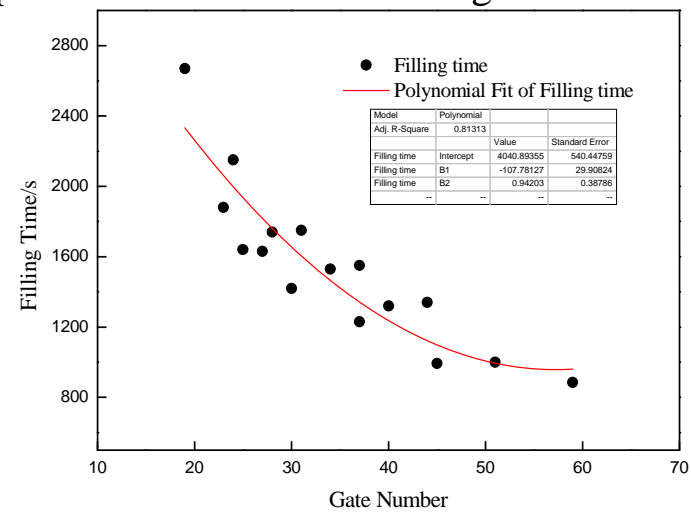

Fig.8 Relationship between gate number and filling time

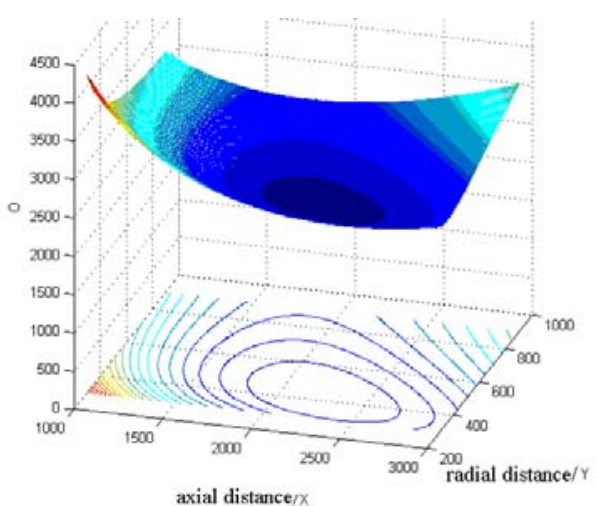

Fig.9 Relationship between optimization objective and tuning parameters

From Fig.9, we can find the optimum parameters are $(X, Y)=(2240,423) \mathrm{mm}$, the filling time on this condition is $1137.9 \mathrm{~s}$, the process cost is 1329.4 , both the constraints (equations 3 ) are satisfied.

\section{Conclusions}

In this paper, the filling stage of VIMP for composite yacht hull was systematically studied by numerical simulation technology in order to provide important foundation for ply design, mold design and process optimization. We analysed the mechanism of air entrapment in filling process, studied the manufacturability evaluation of the ply scheme. We selected the best runner layout pattern based on the filling simulation under different runner layout patterns. The method for gate location optimization was also researched. The mentioned methods were detailed described and verified by the optimization process of a practical yacht. The simulations also showed that it is inadvisable to reduce the filling time just by adding the gate quantity.

\section{References}

[1] George Marsh. Composites- the enabler for large fast yachts[J]. Reinforced Plastics, 12, (2000): 28-31.

[2] W. D. Brouwer, E. C. F. C. van Herpt, M. Labordus. Vacuum injection moulding for large structural applications [J]. Composites: Part A, 34, (2003): 551-558.

[3] Q. Govignon, S. Bickerton, P. A. Kelly. Simulation of the reinforcement compavtion and resin flow during the complete resin infusion process[J]. Composite Part A, 41(2010): 45-57.

[4] N. C. Correia. E. Robitaille, A. C. Long. Analysis of the vacuum infusion moulding process: IAnalytical formulation[J]. Composite: Part A, 2005: 1645-1656.

[5] F. Cappello, A. Mancuso. Lay-up optimization for the hull of a racing sailing yacht[J]. Advances in Engineering Software 32 (2001) : 133-139.

[6] Ali Gokce, Suresh G. Advani. Simultaneous gate and vent location optimization in liquid composite molding processes[J]. Composites: Part A ,35 (2004) : 1419-1432.

[7] Ali Gokce, Kuang-Ting Hsiao, Suresh G. Advani. Branch and bound search to optimize injection gate locations in liquid composite molding processes[J]. Composites: Part A, 33 (2002): 1263-1272. [8] Y.C. Lam, G.A. Britton, D.S. Liu. Optimisation of gate location with design constraints[J]. Int J Adv Manuf Technol, 24 (2004): 560-566.

[9] JIANG Shun liang. New method for RTM gate optimization[J]. ACTA MATERIAE COMPOSITAE SINICA, 19(2002): 18-23. 